\title{
Distribution in microbial genomes of genes similar to lod $A$ and goxA which encode a novel family of quinoproteins with amino acid oxidase activity
}

Jonatan C Campillo-Brocal, María Dolores Chacón-Verdú, Patricia Lucas-Elío and Antonio Sánchez-Amat*

\begin{abstract}
Background: L-Amino acid oxidases (LAOS) have been generally described as flavoproteins that oxidize amino acids releasing the corresponding ketoacid, ammonium and hydrogen peroxide. The generation of hydrogen peroxide gives to these enzymes antimicrobial characteristics. They are involved in processes such as biofilm development and microbial competition. LAOs are of great biotechnological interest in different applications such as the design of biosensors, biotransformations and biomedicine.

The marine bacterium Marinomonas mediterranea synthesizes LodA, the first known LAO that contains a quinone cofactor. LodA is encoded in an operon that contains a second gene coding for LodB, a protein required for the post-translational modification generating the cofactor. Recently, GoxA, a quinoprotein with sequence similarity to

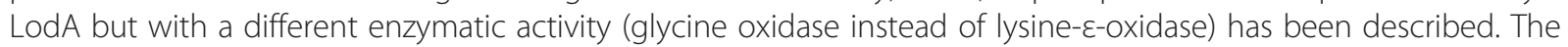
aim of this work has been to study the distribution of genes similar to lodA and/or goxA in sequenced microbial genomes and to get insight into the evolution of this novel family of proteins through phylogenetic analysis.

Results: Genes encoding LodA-like proteins have been detected in several bacterial classes. However, they are absent in Archaea and detected only in a small group of fungi of the class Agaromycetes. The vast majority of the genes detected are in a genome region with a nearby lodB-like gene suggesting a specific interaction between both partner proteins.

Sequence alignment of the LodA-like proteins allowed the detection of several conserved residues. All of them showed a Cys and a Trp that aligned with the residues that are forming part of the cysteine tryptophilquinone (CTQ) cofactor in LodA. Phylogenetic analysis revealed that LodA-like proteins can be clustered in different groups. Interestingly, LodA and GoxA are in different groups, indicating that those groups are related to the enzymatic activity of the proteins detected.

Conclusions: Genome mining has revealed for the first time the broad distribution of LodA-like proteins containing a CTQ cofactor in many different microbial groups. This study provides a platform to explore the potentially novel enzymatic activities of the proteins detected, the mechanisms of post-translational modifications involved in their synthesis, as well as their biological relevance.
\end{abstract}

Keywords: L-amino acid oxidase, Quinone cofactor, Post-translational modification, Lysine oxidase, Glycine oxidase

\footnotetext{
* Correspondence: antonio@um.es

Department of Genetics and Microbiology, University of Murcia, Campus de Espinardo, Murcia 30100, Spain
} 


\section{Background}

L-Amino acid oxidases (LAOs) are enzymes which oxidize amino acids releasing the corresponding ketoacid, ammonium and hydrogen peroxide. They have been found in different microbial groups including bacteria $[1,2]$ although the most studied members of this group are the LAOs present in snake venoms [3]. Enzymes oxidizing amino acids are of great biotechnological interest in many different fields such as the design of biosensors, processes of biotransformation and biomedicine [1]. However, in some cases their use is limited by the difficulties of their recombinant expression [4]. In addition to their biotechnological value, the unraveling of novel metabolic pathways of amino acids is of great interest since, apart from their essential roles in primary metabolism, these pathways are also related to the secondary metabolism in processes such as the synthesis of pigments, antibiotics, etc.

The marine gammaproteobacterium Marinomonas mediterranea synthesizes LodA and GoxA, the first two described LAOs that do not contain FAD but a quinone cofactor. LodA was the first enzyme described with Llysine- $\varepsilon$-oxidase activity [5]. It received a new number by the Enzyme Commission (EC 1.4.3.20). It has been demonstrated recently that the cofactor of LodA is cysteine tryptophylquinone (CTQ) [6]. CTQ cofactor was described for the first time in a quinohemoprotein amine dehydrogenase (QHNDH) [7]. Protein quinone cofactors are generated by post-translational modification of amino acid residues in the protein [8]. In the case of LodA, the modification involves the generation of a quinone from tryptophan 581 and its linking to cysteine 516 . In this regard, it has been demonstrated that LodB, a flavoprotein encoded in the same operon as LodA, is involved in the post-translational modification that generates the quinone cofactor $[9,10]$.

LodA and similar proteins play a role in microbial biofilm development and cell dispersal from the biofilm. This dispersion takes place after cell death of part of the population which is mediated by the hydrogen peroxide released [11]. In the Pseudoalteromonas tunicata autolytic protein AlpP, lysine oxidase activity was observed. However, in other cases such as Chromobacterium violaceum and Caulobacter crescentus, although hydrogen peroxide was released, the substrate of the activity was not reported [11].

Genome sequencing of $M$. mediterranea revealed that it contains two other genes with similarity to $\operatorname{lod} A$. In both cases they are followed by a gene with similarity to $\operatorname{lod} B$ [12]. One of those lodA-like genes codes for GoxA, a quinoprotein with glycine oxidase activity and properties, such as high substrate specificity, clearly different to other flavoproteins with glycine oxidase activity [13]. Those observations suggest that proteins similar to LodA could constitute a reservoir of novel enzymatic activities with potential biotechnological interest. A possibility is that they could oxidize different amino acids, or some structurally related compounds.

The aim of this study was to study the distribution of genes encoding proteins similar to LodA and/or GoxA in sequenced microbial genomes and to get insight into the evolution of this novel family of proteins through phylogenetic analysis. We show that proteins similar to LodA are present in several classes of Bacteria, absent in Archaea and detected only in a small group of fungi of the class Agaromycetes. Those proteins can be clustered in different groups, with LodA and GoxA in distinct groups, indicating that the groups observed may inform the enzymatic activity of the protein clusters.

\section{Results and discussion}

\section{Identification of genes similar to $\operatorname{lod} A$ and/or gox $A$ in microbial genomes}

Using as query the sequence of the two M. mediterranea quinoproteins with amino acid oxidase activity: LodA (accession number AAY33849) [5] and GoxA (accession number ADZ90918) [13], BLASTP search was performed against sequenced microbial genomes deposited in the Integrated Microbial Genomes database as of January 8, 2014. Using GoxA as query and a cut-off limit for the E-value of 1e-10, 170 genes encoding proteins with similarity to it were detected. With LodA a slightly smaller number of genes were observed. Since all of them were included in the former group, those 170 genes were used in further analysis. Two genes were not included in the final analysis because they encode hypothetical proteins which seemed to be truncated. This gave a final selection of 168 genes named in this study as lodA-like genes or belonging to the $\operatorname{lod} A$ family (Additional file 1: Table S1).

Regarding the distribution of genes encoding proteins of the LodA family in microbial genomes it is important to indicate that the number of microbial genomes sequenced show an uneven distribution, with some microbial groups more represented than others (Table 1). For that reason, the number of genomes with genes of the $\operatorname{lod} A$ family is generally expressed in this paper as percentage of the total of microbial genomes sequenced in the group considered. Genes encoding proteins of the LodA family were not detected in Archaea (602 genomes sequenced at the time of the analysis). In Eukarya they have been detected in just 4 strains, with Gymnopus luxurians containing two copies (Table 2). Although the number of strains is low, in terms of percentage, they represent the $1.9 \%$ of the total number of Eukarya sequenced and 26.6\% of the Basydiomycota (Table 1).

Most of the genes encoding proteins of the LodA family were found in Bacteria (Table 1). In this domain they were detected in, approximately, the $0.94 \%$ of the genomes sequenced. In most of the bacterial groups the percentage of bacteria with those genes was around 1-3\%, with some 
Table 1 Distribution of lodA-like genes in microbial genome sequences deposited with IMG database as of January 2014

\begin{tabular}{|c|c|c|}
\hline Taxon & $\begin{array}{l}\text { Genomes with } \\
\text { lodA-like genes }\end{array}$ & Percentage \\
\hline 01 Archaea (602) & 0 & $<0.16$ \\
\hline 01 Bacteria $(14983)^{1}$ & 140 & 0.94 \\
\hline 02 Acidobacteria (34) & 1 & 2.94 \\
\hline 02 Actinobacteria (1401) & 13 & 0.93 \\
\hline 02 Bacteroidetes (598) & 11 & 1.83 \\
\hline 02 Chloroflexi (90) & 2 & 2.22 \\
\hline 02 Cyanobacteria (313) & 9 & 2.88 \\
\hline 02 Firmicutes (3658) & 1 & 0.03 \\
\hline 03 Bacilli (2695) & 1 & 0.04 \\
\hline 04 Bacillales (1103) & 1 & 0.09 \\
\hline 04 Lactobacillales (1592) & 0 & $<0.06$ \\
\hline 03 Clostridia (799) & 0 & $<0.13$ \\
\hline 03 Erysipelotrichia (33) & 0 & $<3.03$ \\
\hline 03 Negativicutes (86) & 0 & $<1.16$ \\
\hline 03 unclassified (45) & 0 & $<2.22$ \\
\hline 02 Planctomycetes (40) & 3 & 7.50 \\
\hline 02 Proteobacteria (7187) & 100 & 1.39 \\
\hline 03 Alphaproteobacteria (1233) & 37 & 3.00 \\
\hline 03 Betaproteobacteria (774) & 19 & 2.45 \\
\hline 03 Deltaproteobacteria (183) & 3 & 1.64 \\
\hline 03 Epsilonproteobacteria (469) & 0 & $<0.21$ \\
\hline 03 Gammaproteobacteria (4466) & 41 & 0.92 \\
\hline 03 Zetaproteobacteria (19) & 0 & $<5.26$ \\
\hline 03 unclassified (43) & 0 & $<2.33$ \\
\hline 01 Eukarya $(203)^{2}$ & 4 & 1.97 \\
\hline 02 Basidiomycota (15) & 4 & 26.66 \\
\hline
\end{tabular}

The numbers in brackets indicate the number of genomes sequenced in each taxon. The number before each taxon is 01 for Domain, 02 for Phylum, 03 for Class and 04 for Order.

${ }^{1}$ Phyla among Bacteria without lodA-like genes: Aquificae (22),

Armatimonadetes (9), Atribacteria (1), Caldiserica (2), Candidatus Saccharibacteria (5), Chlamydiae (100), Chlorobi (25), Chrysiogenetes (2), Deferribacteres (7), Deinococcus-Thermus (43), Dictyoglomi (2), Elusimicrobia (3), Fibrobacteres (9), Fusobacteria (47), Gemmatimonadetes (8), Lentisphaerae (3), Nitrospinae (1), Nitrospirae (19), Poribacteria (11), Spirochaetes (414), Synergistetes (20), Tenericutes (146), Thermodesulfobacteria (6), Thermotogae (40), Verrucomicrobia (34), Candidate division CD12 (1), Candidate division EM 3 (2), unclassified (680).

${ }^{2}$ Phyla among Eukarya without lodA-like genes: Apicomplexa (12), Ascomycota (77), Bacillariophyta (2), Blastocladiomycota (1), Chlorophyta (8) Chytridiomycota (2), Microsporidia (5), Neocallimastigomycota (4), unclassified (26).

exceptions. In the case of Firmicutes, out of 3658 genomes there was a single genome identified (Paenibacillus pinihumi) containing a lodA gene. Two groups of microorganisms with a high number of genomes sequenced but with no lodA-like genes were Spirochaeta and Tenericutes (414 and 146 genomes respectively). Most of the $\operatorname{lod} A$ family genes were detected in Proteobacteria (121 genes out of
168). However, this seems to be the result of the high number of genomes sequenced in this group, since the percentage of Proteobacteria with $\operatorname{lod} A$ genes is on the average (1.39\%). Among Proteobacteria, they were most abundant in Alpha and Betaproteobacteria (3 and 2.45\% respectively). They were not found in Epsilonproteobacteria (0 out of 469 genomes). The percentage in Gammaproteobacteria was $0.94 \%$.

According to their phylogenetic distribution, lodA-like genes seem to have an ancient origin since they are detected in a wide range of bacteria. In contrast, they are detected in a small number of fungi which are phylogenetically related since all of them belong to the class Agaromycetes. A possible explanation for this distribution could be that the acquisition of the gene in fungi took place through a process of horizontal gene transfer from a bacterium. The phylogenetic analysis performed in this study (see below) does not make it possible to suggest the bacterial origin of the fungal genes since the most similar proteins belong to different bacterial groups.

The 168 genes selected were distributed in 144 different microbial genomes since several microorganisms contained more than one copy (Table 2). Only two marine gammaproteobacteria, Marinomonas mediterranea MMB-1, order Oceanospirillales, and Pseudoalteromonas citrea, order Alteromonadales, showed three copies of those genes. In fact, genes of the $\operatorname{lod} A$ family are common in both genera. 4 out of the 5 Marinomonas genomes sequenced contained this kind of genes, although only $M$. mediterranea contained more than one copy. In the genus Pseudoalteromonas, about $50 \%$ of the strains sequenced showed genes of the lodA family. Moreover, 6 out of 8 of those genomes showed two or more copies.

Interestingly, no known human or animal pathogen contains genes of the $\operatorname{lod} A$ family. On the contrary, many of the microorganisms with that kind of genes have been isolated from the microbiota of plants or interact with them. For example, this is the case of many symbiotic Alphaproteobacteria such as Bradyrhizobium. In Gammaproteobacteria there are also many examples of microorganisms associated with algae or plants such as $M$. mediterranea, which is a member of the microbiota of the seagrass Posidonia oceanica [14]. These observations suggest the possibility of an ecological role of this kind of enzymes in the interaction between the plant and its associated microbiota, or in the growth of the microorganisms on the surface of the plant. In this regard, it has been observed that in several microorganisms these LodA-like proteins are involved in biofilm differentiation and dispersal [11].

\section{Detection of lodB-like genes in genomes with genes of the $\operatorname{lod} A$ family}

lod and gox operons contain, immediately downstream to the gene coding for the protein of the LodA family, a 
Table 2 Microbial genomes deposited with IMG as of January 2014 with more than one copy of genes encoding proteins similar to LodA/GoxA

\begin{tabular}{lllll}
\hline Genome Name & Phylum & Class & Phylogenetic Group of LodA-like proteins \\
\hline Tenacibaculum ovolyticum DSM 18103 & Bacteroidetes & Flavobacteria & II & IIIA \\
Kordia algicida OT-1 & Bacteroidetes & Flavobacteria & II & IVB \\
Thalassobaculum salexigens DSM 19539 & Proteobacteria & Alphaproteobacteria & IIB & IIB \\
Bradyrhizobium japonicum USDA 38 and USDA 6 & Proteobacteria & Alphaproteobacteria & ID & IVA \\
Nitrobacter hamburgensis X14 & Proteobacteria & Alphaproteobacteria & ID & None \\
Xanthobacter sp. 126 & Proteobacteria & Alphaproteobacteria & IB & None \\
Citreicella sp. SE45 & Proteobacteria & Alphaproteobacteria & IB & IIIB \\
Burkholderia sp. BT03 & Proteobacteria & Betaproteobacteria & III & None \\
Chitinimonas koreensis DSM 17726 & Proteobacteria & Betaproteobacteria & ID & III \\
Cellvibrio japonicus Ueda107 & Proteobacteria & Gammaproteobacteria & IB & III \\
Marinomonas mediterranea MMB-1 & Proteobacteria & Gammaproteobacteria & IA & IIB \\
Oceanospirillum beijerinckii DSM 7166 & Proteobacteria & Gammaproteobacteria & IB & III \\
Pseudoalteromonas citrea NCIMB 1889 & Proteobacteria & Gammaproteobacteria & IA & II \\
Pseudoalteromonas luteoviolacea 2ta16 & Proteobacteria & Gammaproteobacteria & IA & IB \\
Pseudoalteromonas rubra ATCC 29570 & Proteobacteria & Gammaproteobacteria & IB & IIIA \\
Pseudoalteromonas flavipulchra 2ta6, JG1 & Proteobacteria & Gammaproteobacteria & IA & IIIA \\
Pseudoalteromonas piscicida ATCC 15057 and JCM 20779 & Proteobacteria & Gammaproteobacteria & IA & IIIA \\
Rheinheimera sp. A13L & Proteobacteria & Gammaproteobacteria & IA & IIIA \\
Gymnopus luxurians FD-317 M1 & Basidiomycota & Agaricomycetes & V & V \\
\hline
\end{tabular}

second gene coding for a putative flavoprotein (Figure 1A). In the lod operon this protein was named LodB and it has been demonstrated that it is required for the posttranslational modification generating active LodA $[9,10]$. In all but one of the genomes analyzed, similarly to lod and gox operons, it has been possible to detect downstream the $\operatorname{lod} A$-like gene, or very close in the genome, a lodB-like gene showing the conserved COG0644 described in flavoproteins (Additional file 2: Table S2).

The only genome in which no lodB-like gene could be detected close to the lodA-gene was Cryptosporangium arvum. In two other cases, the lack of the lodB-like gene could be accidental. In relation to the $\operatorname{lod} A$-like gene CSE45_2361 from Citreicella sp. SE45, there is a large intergenic region downstream that gene. The examination of this sequence allowed the detection in direction 3' -5 ' of a non-annotated ORF which codes for a protein bearing similarity to LodB-like proteins. In Nitrolacentus hollandicus LD the genome sequencing was incomplete and the lodA-like gene is at the end of a contig, so it was not possible to locate the lodB-like gene. However, a gene in another contig with accession number 2520384730 is a good candidate.

There are some modifications to the general pattern of a $\operatorname{lod} B$-like gene located downstream the $\operatorname{lod} A$-like gene. For instance, in 10 genomes (four of them Bradyrhizobium) lodB-like genes are located downstream to the
lodA-like gene but separated from it by an additional small gene with a size between 138-142 amino acids (Additional file 2: Table S2 and Figure 1B). These genes code for small hypothetical proteins showing higher than $50 \%$ amino acid identity between them. These proteins do not possess any conserved domain. Since, as far as we know, no protein of this group has been characterized, their function remains unknown. Interestingly, the genomic organization suggests that all of the operons containing those small genes also include other genes coding for hypothetical tyrosinases and multicopper oxidases (Figure 1B). M. mediterranea also synthesizes tyrosinase and multicopper oxidase enzymes which are co-regulated with LodA, although they are not encoded by genes located in the same operon [16]. These observations suggest some possible functional relationship between all those enzymes.

With regard to exceptions to the general organization discussed above, in Acidovorax avenae RS-1 and in one lodA-like gene of Oceanospirillum beijerinckii DSM7166 (H579DRAFT_00201), the lodA-like genes are followed by two genes with similarity to flavoproteins. The comparison of these proteins with LodB revealed that the first one shows high similarity to the $\mathrm{N}$-terminal region of LodB, while the second one is similar to the Cterminal region. For example, the product of Oceanospirillum beijerinckii DSM7166 gene H579DRAFT_00202 


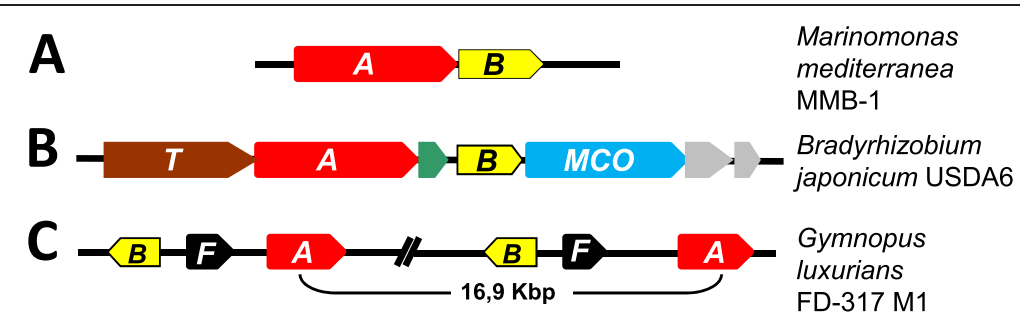

Figure 1 Genome organization around the lodA-like genes ( $A$ in red) and lodB-like genes ( $B$ in yellow) in selected genomes. $A$ ) lod operon in M. mediterranea [15]. B) Bradyrhizobium japonicum USDA6 operon containing lodA and lodB-like genes separated by a small gene shown in green. The gene in brown (marked with a T) encodes a protein containing the domain pfam00264 (tyrosinases). The product of the blue gene contains the domains pfam07731 and pfam07732 characteristic of multicopper oxidases (MCO). C) Gymnopus luxurians FD-317 M1 genome organization showing the two copies of lodA and lodB-like genes. The genes in black (marked with an F) encode proteins with the bacterial Ferritin-like domain (PF12902).

showed $47 \%$ similarity to residues $4-118$ of LodB and the product of H579DRAFT_00203 showed 42\% similarity to residues $130-160$ of LodB. These observations suggest as the most likely explanation that those lodB-like genes are the result of the division of a previous gene.

In the five fungal $\operatorname{lod} A$-like genes, the $\operatorname{lod} B$-like genes were located in the opposite orientation. It is also worth mentioning that in these cases a gene coding for a ferritin-like protein was also detected close in the genome or between them (Figure 1C). Apart from fungi, in this study it has been also detected that $\operatorname{lod} A$ and $\operatorname{lodB}$ like genes are in opposite orientations in the genome of the actinobacterium Actinoplanes globisporus.

The observation of the conservation of lodB-like genes associated to $\operatorname{lod} A$-like genes in all but one of the genomes analyzed, suggests a strong selective force to maintain that association. Results from our group showed that each LodB-like protein might specifically be involved in the post-translational modification of its partner protein [17].

\section{Sequence analysis of proteins of the LodA family}

In terms of sequence similarities, the alignment of the proteins similar to LodA detected in this study revealed several conserved residues in all of them (Figure 2). Interestingly, LodA C516 and W581, which have been described as the residues post-translationally modified to generate the quinone cofactor cysteine tryptophylquinone [6], aligned with Cys and Trp in all of the LodAlike proteins, suggesting that they possess the same quinone cofactor. In fact, modeling of GoxA supports that this protein contains CTQ [17]. The other residues conserved in these proteins could be involved in common processes to all of those proteins, being a possibility that they are involved in the generation of the quinone cofactor or in the catalytic activity of the enzymes.

Most of the proteins of the LodA family showed a size between 600 and 700 amino acids (Additional file 1: Table S1). In fact the average size is 738 amino acids, which is very close to the 726 amino acids of LodA. However, there are proteins with only 481 amino acids, such as A3CEDRAFT_0690 from Amycolatopsis balhimycina DSM 44591, and others with much larger sizes such as YY3DRAFT_04971 from Rhizobium sp. STM6155 with 1413 amino acids.

The proteins with larger sizes seem to be the result of gene fusions since, in addition to the sequence characteristics of the LodA family, they show conserved domains described in other proteins (Table 3) [18]. Some of these proteins' $\mathrm{N}$-terminal regions have similarity to the conserved domain pfam00199 characteristic of catalases. Those sequences also match the Conserved Domain CD08152 described in protein families related to the uncharacterized y4iL protein of Rhizobium sp. NGR234 which share the catalase fold and bind to heme, although they do not necessarily have catalase activity [19]. Five other proteins show in their $\mathrm{N}$-terminal region sequences with similarity to the conserved domain pfam14518, described in iron-containing redox enzymes. Finally, two proteins from the actinobacteria Amycolatopsis vancoresmycina and Cryptosporangium arvum show the von Willebrand factor type A (vWA) domain (pfam13519). This domain was first described in the blood coagulation protein von Willebrand factor (vWF) but it has been described in other proteins. It is involved in different cellular processes that imply surface interactions mediated by a metal ion dependent adhesion site termed as the MIDAS motif [20]. The determination of the relevance of those additional conserved domains in proteins of the LodA family will require experimental work aimed at characterizing those proteins.

\section{Phylogenetic analysis of LodA-like proteins}

Once aligned, LodA-like proteins were subjected to phylogenetic analysis using Neighbor-Joining (NJ) and Maximum Likelihood (ML) methods available in the MEGA program [21]. In this study we have clustered the LodA-like proteins in several groups that met the criterion of being supported by bootstrap values higher than $70 \%$ in both phylogenetic analyses. As shown in Figure 3, five groups that included most of the proteins could be 


\begin{abstract}
1 MALSVHPSIG VARLGNANTD NFVLNPMEIG GLPYEHDVDL KPTTTVVNFK DEAGCIRRQG
61 QVFKVFGASN EELTLDSPNV KNIEWTVHLA NKKAAWYEFR ELNGNLLYGR DNSYSARGVP

121 WRNASKTASS ERQSLIIDLG PRSVSGVMAT VEISINNIPE TYLHPSYPSG ELLQGSKHFE

181 SLGTLRTDSQ GRLIVLGGYG FAGGNTDLSG YGGGDDWYDD ISDGSVTCVV TYSDDSSETS

241 TAWMVVGSPD FAPEIVNIST LSDTCFDVGV RNFDLVPDMY DSATGHYKSD YVANEDRDIL

301 PIIQRISQYQ WVSNVQSMSG FFSFQFDYRD GSAANKANRM KYYNYFRQLD NKVIGDYDQP

361 QQVLMSSEVE GDILPLMPMN SGSNSVSSSN FYDLTDNVVE KFLALDATQL FLLGQWAEGE

421 FTAGPADDYP VSDMDTASIG NCVGLPMCPG IEMTWSLQNP VIYKDAYQIK HYQDKAYFDV

481 NGLTPERDEC EEETGCEPGD LTKRMACPWQ ADFFNCTIQT VNFSEPSVNK ASQTETVTSR

541 THYEWGNLPA GVSVPDQSSV SATKNVDEKV PLPPAYYSYW WPPQSPWDVL TGELDTEGQL

601 HSHLPAGQQI NYARGINSYS QMVEHWSALA FIRDRNQNND GFPFFTETER NHELFDFKEV

661 LVGQVTGNSE DNETSLPVFF INANKESLEG KGTKKGKLMA SYFEERAFSK VRSSNIRPRS

721 GTRMRG
\end{abstract}

Figure 2 Sequence analysis of LodA-like proteins. Marked on the sequence of LodA the residues conserved in 100\% of the proteins are shown highlighted in green. Of those, the two underlined are the residues that are modified to generate the cofactor. Other residues conserved in more than $90 \%$ of the selected proteins are shown in red. Several domains proposed to be conserved in LodA-like proteins are highlighted in grey.

established. With the criterion used, other proteins could not be clustered (Figure 3). Among those are included the proteins synthesized by the actinobacteria Amylocatopsis vancoresmycina and Cryptosporangium arvum that show the conserved domain pfam13519 (vWA domain) previously mentioned. In the next paragraphs the characteristics of the different groups will be discussed.

\section{Group I}

Group I contains a total of 56 proteins and could be divided in several subgroups as shown in Figure 3. Group IA contains $M$. mediterranea LodA, the first described enzyme with lysine- $\varepsilon$-oxidase activity [5], as well as $P$. tunicata AlpP in which lysine- $\varepsilon$-oxidase activity has been also demonstrated [11] (Figure 4). Both LodA and AlpP were initially described as antimicrobial proteins $[22,23]$. Interestingly, group IA includes proteins from many microorganisms, many of them in the genus Pseudoalteromonas, for which the synthesis of antimicrobial proteins has been reported. In P. flavipulchra JG1 two genes of the $\operatorname{lod} A$ family are detected, one of them (UY7DRAFT 03653) belongs to the group IA. It was demonstrated that the product of this gene is the protein PfaP which is an antimicrobial protein with high similarity to AlpP and LodA [24]. In another strain of the same species an antimicrobial protein was detected with sequence similarity to PfaP. It showed L-amino acid oxidase activity, but in this case it was able to oxidize not only L-lysine but also other amino acids such as L-Met, Gln, Leu, etc. [25]. An L-amino acid oxidase with similar broad substrate range (Met, Gln, Leu, Phe, Glu, Trp, etc.) has been described in several $P$. luteoviolacea strains whose genome has not been sequenced yet [26]. At the time of preparation of this manuscript there were two $P$. luteoviolacea strains whose genome had been sequenced. Strain B did not show any gene of the lodA family, although it is important to bear in mind that it has been proposed that this strain was misclassified and should be placed in a different species [27]. In strain 2ta16, two genes were detected, one in the group IA and the second in the group IB. As far as we know, no antimicrobial activity has been reported in this strain. Accordingly, for this species it is not possible to establish a relationship between the antimicrobial proteins and the lodA-like genes detected. With regards to other genera, in Rheinheimera aquatica strain GR5 lysine oxidase activity was demonstrated for a protein with a peptide fragment with high similarity to LodA and AlpP [28,29]. That fragment is also similar to the product of the gene Rhein1334 belonging to group IA from the sequenced Rheinheimera sp. strain A13L (Figure 4). The existence of this gene has been previously reported indicating that it could encode a lysine- $\varepsilon$-oxidase [30]. Interestingly this strain contains a second lodA-like gene that is phylogenetically distant (group III). Overall, our results indicate that proteins in the group IA show L-amino acid oxidase activity and that this capacity confers to them antimicrobial properties.

Apart from group IA, with the exception of the gene of the deltaproteobacterium Corallococcus coralloides, the other genes in group I could be associated to different clusters (IB, IC and ID) (Figure 3, Additional file 3: Figure S1 and Additional file 4: Figure S2). Group ID (Additional file 4: Figure S2) is particularly interesting since it contains the operons previously mentioned in which the lodA-like gene is separated from the lodB-like gene by a small gene (Figure 1B). Saccharophagus degradans $2-40$ is the only microorganism in this group in which there is no small gene between lodA-like and 
Table 3 Products of the lodA-like genes showing conserved domains in addition to the characteristic sequences of the LodA family of proteins

\begin{tabular}{|c|c|c|c|c|}
\hline Genome & gene_oid & Locus Tag & AA Seq Length & Pfams \\
\hline Actinoplanes globisporus DSM 43857 & 2515244410 & A3CQDRAFT_07977 & 985 & pfam14518 \\
\hline Burkholderia sp. BT03 & 2536908549 & PMI06_03990 & 1409 & pfam 14518 \\
\hline Calothrix sp. PCC 7103 & 2507474092 & Cal7103DRAFT_00009910 & 1049 & pfam14518 \\
\hline Paenibacillus pinihumi DSM 23905 & 2524187775 & H583DRAFT_01923 & 1099 & pfam 14518 \\
\hline Rhizobium sp. STM6155 & 2513599306 & YY3DRAFT_04971 & 1413 & pfam14518 \\
\hline Acinetobacter gyllenbergii MTCC 11365 & 2546621803 & L293_0743 & 1008 & pfam00199 \\
\hline Acinetobacter sp. NBRC 100985 & 2533901541 & & 1008 & pfam00199 \\
\hline Acinetobacter tjernbergiae DSM 14971 & 2518262899 & C502DRAFT_01575 & 1006 & pfam00199 \\
\hline Azospirillum lipoferum 4B & 2512035869 & AZOLI_p50417 & 999 & pfam00199 \\
\hline Azospirillum sp. B510 & 646556648 & AZL_e04100 & 1004 & pfam00199 \\
\hline Burkholderia sp. BT03 & 2563064361 & PMI06_008734 & 963 & pfam00199 \\
\hline Cupriavidus sp. UYPR2.512 & 2514031881 & A3A5DRAFT_06866 & 1025 & pfam00199 \\
\hline Flavobacterium soli DSM 19725 & 2523123554 & G508DRAFT_03147 & 1072 & pfam00199 \\
\hline Massilia timonae CCUG 45783 & 2532942463 & HMPREF9710_03282 & 979 & pfam00199 \\
\hline Microcystis aeruginosa PCC 9701 & 2535024168 & & 990 & pfam00199 \\
\hline Oceanospirillum beijerinckii DSM 7166 & 2524095414 & H579DRAFT_00201 & 973 & pfam00199 \\
\hline Pseudoalteromonas rubra ATCC 29570 & 2541428757 & PRUB_24676 & 1039 & pfam00199 \\
\hline Pseudoalteromonas sp. BSi20495 & 2540458794 & & 1000 & pfam00199 \\
\hline Pseudoalteromonas sp. Bsw20308 & 2540452162 & D172_1358 & 1000 & pfam00199 \\
\hline Ralstonia solanacearum MolK2 & 2541798314 & & 1000 & pfam00199 \\
\hline Ralstonia solanacearum Po82 & 651230827 & RSPO_m00447 & 999 & pfam00199 \\
\hline Rhizobium leguminosarum bv. viciae 128C53 & 2515651856 & B062DRAFT_04548 & 1004 & pfam00199 \\
\hline Sphingomonas sp. S17 & 651582060 & SUS17_588 & 986 & pfam00199 \\
\hline Streptomyces afghaniensis 772 & 2546772914 & STAFG_1983 & 999 & pfam00199 \\
\hline Streptomyces purpureus KA281, ATCC 21405 & 2516519010 & StrpuDRAFT_3616 & 993 & pfam00199 \\
\hline Tenacibaculum ovolyticum DSM 18103 & 2523672835 & H518DRAFT_02976 & 1061 & pfam00199 \\
\hline Amycolatopsis vancoresmycina DSM 44592 & 2546378692 & H480_25957 & 1114 & pfam13519 \\
\hline Cryptosporangium arvum YU 629-21 & 2510402938 & CryarDRAFT_3973 & 1026 & pfam13519 \\
\hline
\end{tabular}

lodB-like. Operons encoding proteins of group ID also contained genes encoding hypothetical tyrosinases and multicopper oxidases. The possible relationship between these two copper oxidases with LodA-like proteins is noteworthy since $M$. mediterranea also synthesizes a tyrosinase and a multicopper oxidase, distantly located in the genome, which are co-regulated with LodA [16]. In any case, the similarity between the operons in group ID and the fact that they are present in microorganisms in different taxonomic groups, most of them Alphaproteobacteria $(7 / 11)$ but it also includes 1 Acidobacteria, 1 Gammaproteobacteria, 1 Betaproteobacteria and 1 Bacteroidete (Additional file 4: Figure S2), strongly suggests some kind of functional conservation of the enzymes encoded by all those genes.

In relation to the possible enzymatic activity of the proteins of groups IB, IC or ID, no oxidase activity has been reported for any of them. However, it has been shown that the proteins from Caulobacter crescentus (group IB) and Chromobacterium violaceum (group IC) are involved in hydrogen peroxide generation during biofilm development by those microorganisms [11]. Systematic analysis did not allow the detection of lysine oxidase activity in the cultures of those microorganisms as well as in Marinomonas sp. MED121 (IB) and Saccharophagus degradands 2-40 (ID) (Campillo-Brocal et al., unpublished observations). These data suggest that these enzymes oxidize substrates different to L-lysine.

In terms of taxonomic distribution, Proteobacteria in general and Gammaproteobacteria in particular are highly represented in group I. Gammaproteobacteria constitute the $71.4 \%$ (15 out of 21 ) of the microorganisms in the subgroup IA (Figure 4) and 68.4\% (13/19) in group IB (Additional file 3: Figure S1). In contrast, they 


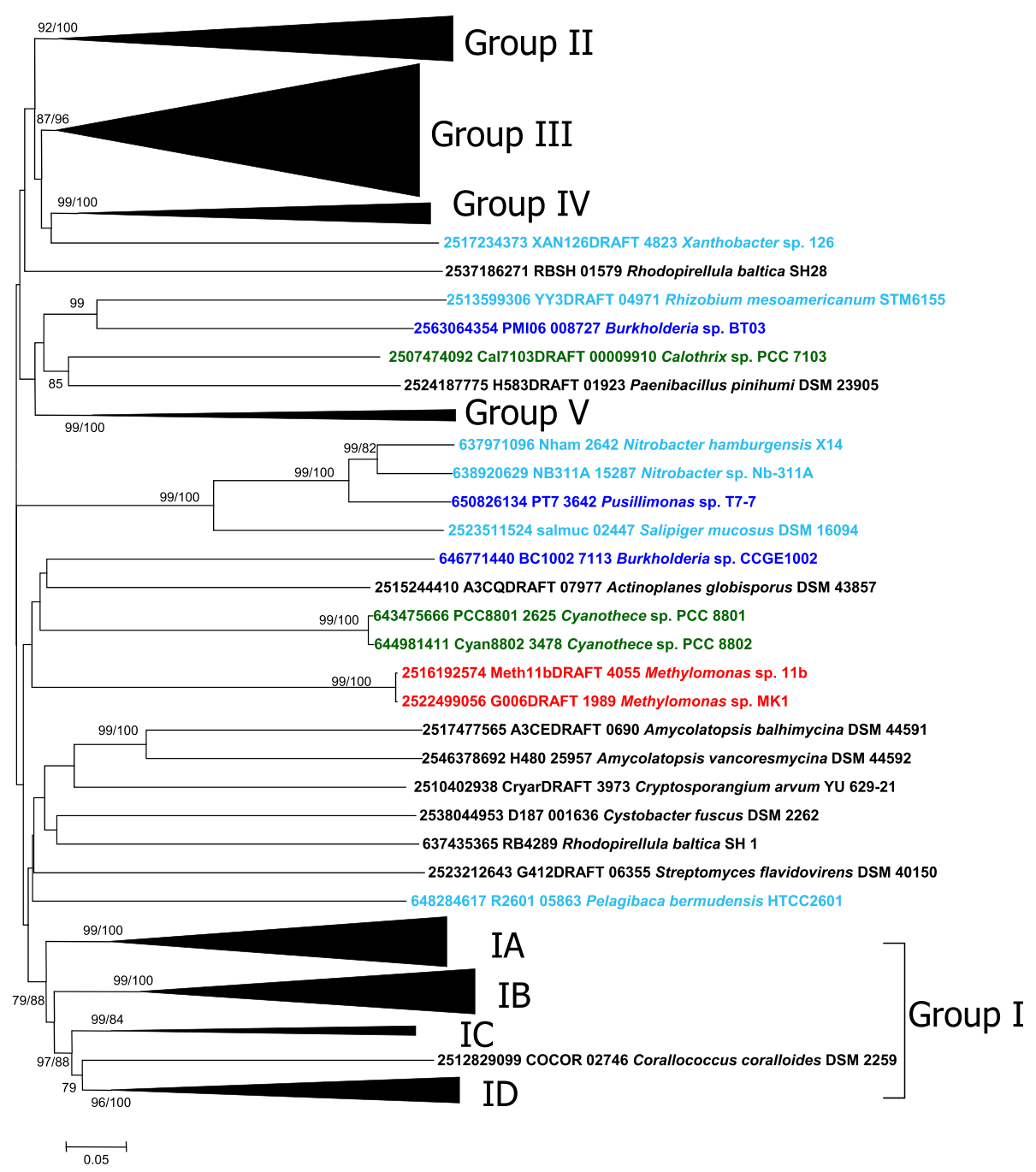

Figure 3 Phylogenetic relationships of LodA-like proteins. The tree was constructed using the Neighbor-Joining method built in the MEGA6 program. The distances between the proteins were computed using the p-distance method and are in the units of the number of amino acid differences per site. Numbers at branches indicate bootstrap values higher than 70\% for both Neighbor-Joining and Maximum Likelihood trees. The nonclustered Gammaproteobacteria are indicated in red, Alphaproteobacteria in light blue, Betaproteobacteria in dark blue and photosynthetic microorganisms in green.

are the $28.4 \%$ (41/144) of the total number of microorganisms with genes of the lodA family. Interestingly, no Alphaproteobacteria is included in group IA and only two belong to group IB, but they are abundant in group ID (7/10). Group IC include microorganisms belonging to different taxonomic groups (Additional file 4: Figure S2).

\section{Group II}

GoxA, a glycine oxidase synthesized by Marinomonas mediterranea [13] clusters in group II of LodA-like proteins (Figure 5). The fact that two M. mediterranea proteins, LodA and GoxA, with different enzymatic activities, belong to different groups indicates that the phylogenetic groups described in this study are of interest for exploring the enzymatic activity of the LodA-like proteins. All the proteins in group II, except for the proteins from Kordia algicida,
Tenacibaculum ovolyticun and Pseudoalteromonas citrea, could be subdivided in two groups (IIA and IIB) (Figure 5). In terms of protein sequence, an important difference is that the three nonclustered proteins do not show in their $\mathrm{N}$-terminal region a typical twin arginine secretion motif present in all of the others.

It is important to point out that most (13/15) of the proteins of groups IIA and IIB were detected in genomes of Alphaproteobacteria. The only two that did not belong to this Class are the betaproteobacterium Alcaligenes faecalis subsp. phenolicus and the gammaproteobacterium Marinomonas mediterranea. In terms of sequence comparison, according to EMBOSS-Needle [31], LodA and GoxA showed $22.8 \%$ identity and $34.6 \%$ similarity, while GoxA and the LodA-like protein of Nisaea denitrificans showed 64.5\% identity and $76.4 \%$ similarity. These results suggest that $M$. 


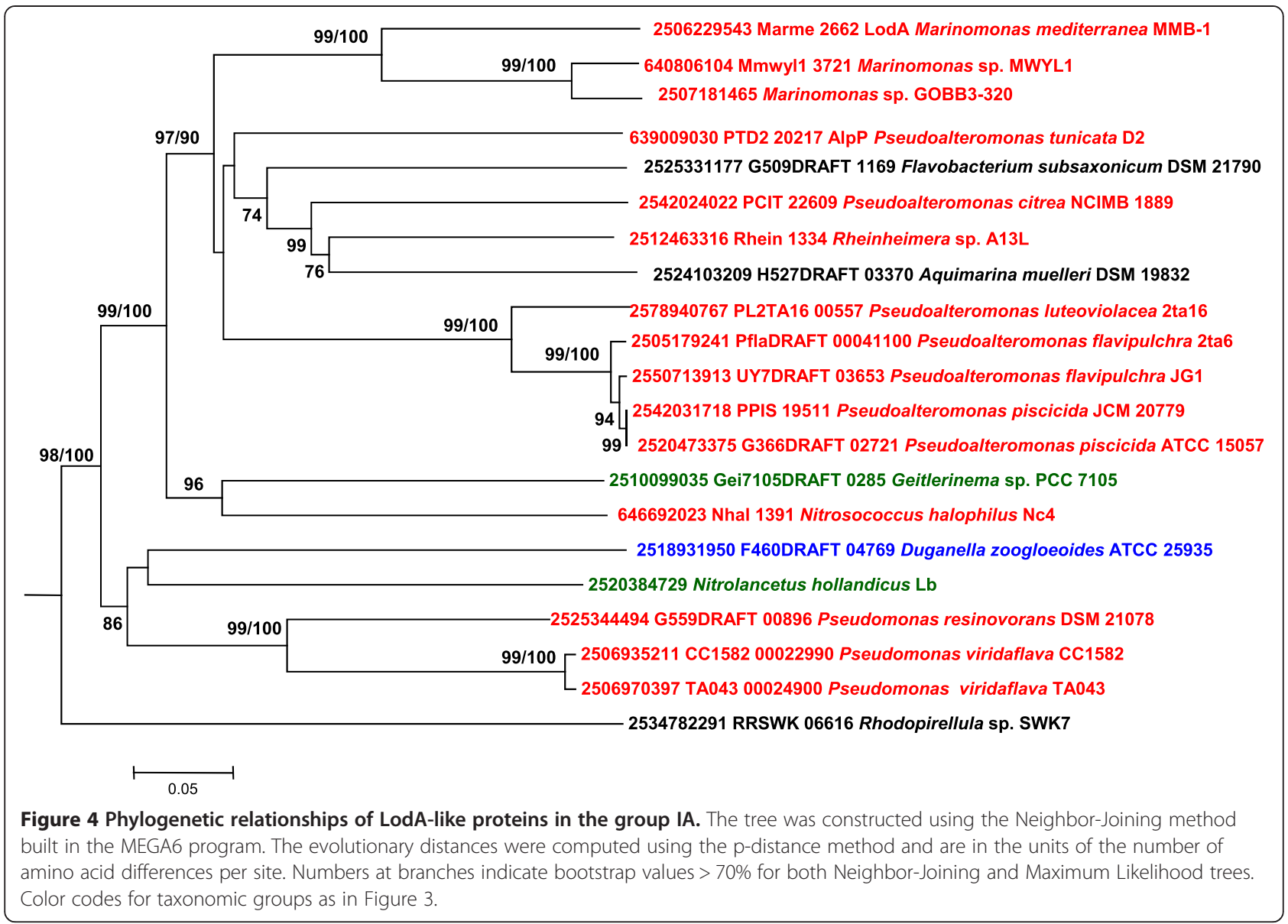

mediterranea could have acquired the gox $A$ gene through a process of horizontal gene transfer from an alphaproteobacterium rather than by gene duplication. A process of horizontal gene transfer could have also generated the lodA-like gene in Alcaligenes faecalis subsp. phenolicus, the only betaproteobacterium in this group (Figure 5). In contrast, Thalassobaculum salexigens contains two copies of $\operatorname{lod} A$-like genes which seem to have been generated by gene duplication, since the two proteins synthesized by this microorganism show 54.8 identity and 66.5 similarity, and cluster together very close in group II (Figure 5).

\section{Group III}

Group III of LodA-like proteins include a wide range of proteins detected in different microbial groups (Additional file 5: Figure S3). It was possible to detect some subgroups of proteins in group III. Group IIIA contains many proteins synthesized by Gammaproteobacteria, while group IIIC only contains Actinobacteria. Group III includes all the proteins detected in this study with a fusion to the domain pfam00199 (the catalase domain above mentioned) as well as many other proteins that do not show that fusion. The proteins with domain pfam00199 did not cluster in any subgroup in particular.
As far as we know, no protein in this group has been characterized. Group III includes the product of Marme_2396 which is the third gene of the lodA family detected in Marinomonas mediterranea. As it has been previously described, the other two genes encode a lysine- $\varepsilon$-oxidase and a glycine oxidase. A double mutant with deletion of lod and gox operons lost both activities suggesting that the protein in group III shows a different enzymatic activity [13].

In many microorganisms in which more than one copy of lodA-like genes have been detected, one of those copies belonged to group III (Table 2). For instance, Pseudoalteromonas citrea and $M$. mediterranea were the only two bacteria detected with three copies of genes of the $\operatorname{lod} A$ family. Interestingly, in both cases the three copies were in the groups I, II and III as defined in this study. This observation suggests that the function of the proteins encoded by those genes could be complementary, perhaps acting on different substrates.

\section{Group IV}

Group IV is a small group containing just 9 members (Additional file 6: Figure S4). Two subgroups of proteins could be recognized in it. Subgroup IVA contains 


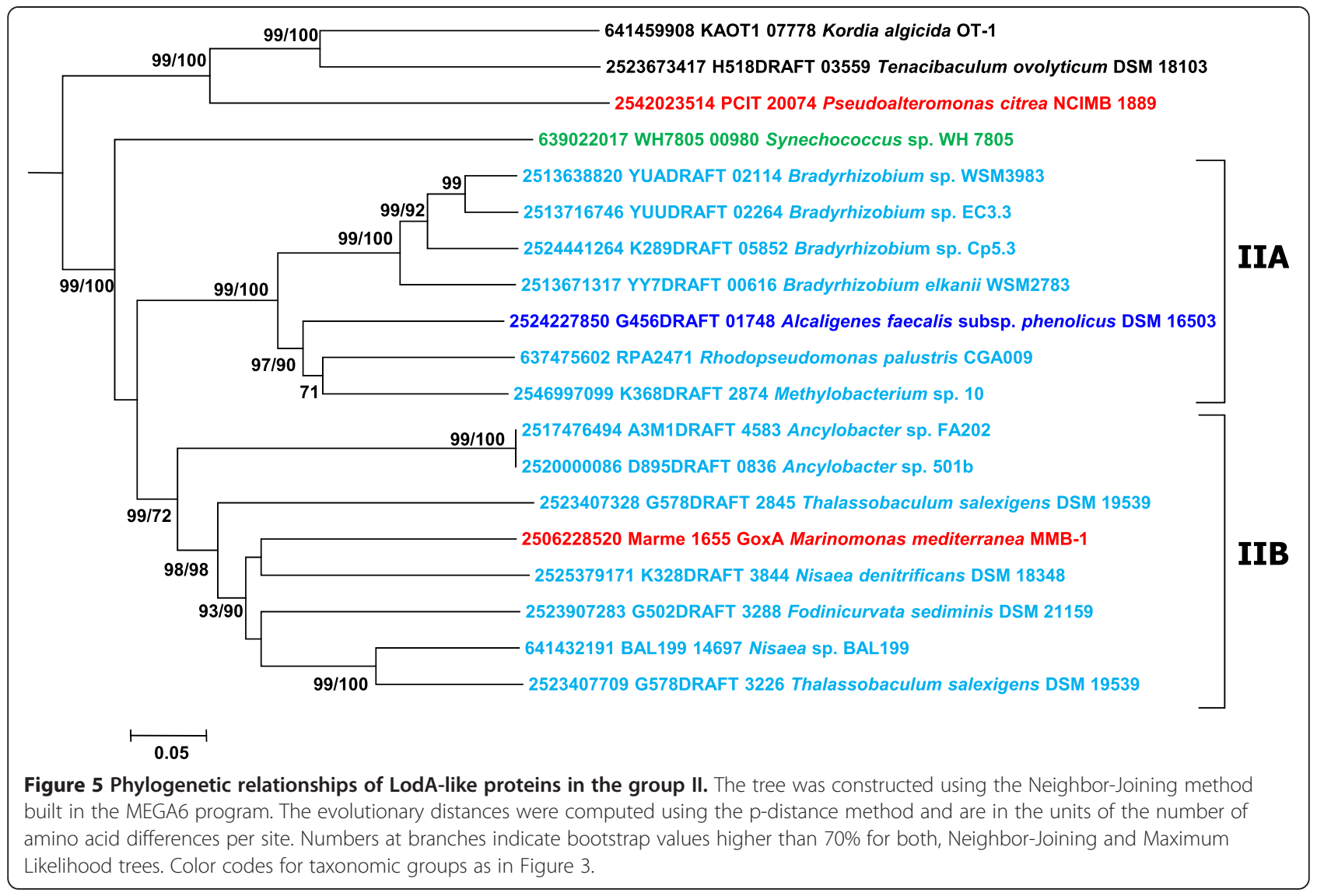

proteins synthesized by Alphaproteobacteria of the order Rhizobiales. In subgroup IVB proteins of several bacterial Classes including Flavobacteria, Deltaproteobacteria, and Actinobacteria were included. No protein of this group IV has been characterized so far.

\section{Group V}

This small group contains the five proteins whose encoding genes were detected in fungi, including the two copies detected in Gymnopus luxurians (Additional file 7: Figure S5). These two proteins showed higher similarity between them (60.2\% identity and $70.5 \%$ similarity) than to any other LodA-like protein detected in this study. In addition, the genes encoding those proteins are close in the fungal genome (Figure 1C). These observations suggest a possible genetic duplication event. As previously discussed, the lodA-like genes detected in fungi show an unusual genetic organization since the $\operatorname{lod} B$-like gene is oriented in the opposite direction to the lodA gene (Figure 1C). In terms of sequence analysis, the LodA-like proteins synthesized by fungi do not show defining features being very similar to the bacterial proteins.

\section{Conclusions}

LodA and GoxA are unconventional amino acid oxidases since they are the first enzymes of this group whose cofactor is CTQ and not FAD $[5,6,13]$. Genome mining using the sequences of the M. mediterranea LodA and GoxA have revealed the presence of 168 genes encoding proteins similar to these two in 144 microbial genomes, representing the $0.91 \%$ of all the genomes deposited in IMG database as of January 2014. Many of those genes were annotated as encoding hypothetical proteins, although since the description of LodA, some of them are reported as encoding lysine oxidases. However, as this study reveals, LodA-like proteins can be divided in several clusters and the enzymatic activity may depend on the group considered. For example, $M$. mediterranea GoxA does not show lysine oxidase but glycine oxidase activity [13].

This study provides a platform to analyze the enzymatic activity of novel LodA-like proteins which we consider to be a reservoir of novel enzymatic activities of potential biotechnological interest. Moreover, this study will be very helpful to experimentally address structurefunction studies in LodA-like proteins. For example, sequence analysis has revealed several conserved domains and residues in all the proteins analyzed. The functions 
of most of those domains and residues remain to be analyzed. However, it seems plausible to consider that the conservation of a $\mathrm{C}$ and $\mathrm{W}$ that align with the $\mathrm{C}$ and $\mathrm{W}$ in LodA CTQ cofactor indicates that this cofactor is present in all of the proteins detected.

In relation to cofactor biosynthesis, it has been observed that even when a microorganism shows more than one copy of a lodA-like gene, each copy is generally followed by a copy of a lodB-like gene. This observation suggests that there is a very specific interaction between LodA-like proteins and the flavoproteins encoded in the same operon. In the case of M. mediterranea LodA, it has been shown that LodB participates in the generation of the quinone cofactor [9]. Recent studies of our group indicate that in the generation of the cofactor there is a specific interaction between the flavoprotein and the quinoprotein encoded in the same operon [17].

LodA-like proteins seem to have an ancient origin in bacteria since they are present in many different groups. Their evolution in bacterial genomes seems to involve different processes. The data obtained suggest that in some cases, such as the presence of goxA in M. mediterranea, a process of horizontal gene transfer could have been involved. While in others, such as T. salexigens, a genetic duplication event seems to be the most reasonable explanation.

\section{Methods}

\section{Detection of lodA-like and lodB-like genes}

Most of the analysis performed in this study have been carried out using the tools available at Integrated Microbial Genomes Expert Review (IMG/MER) [32]. Genes encoding proteins similar to LodA with an E-value lower than 1e-10 were identified using BLASTP search using as query the sequences of LodA (accession number AAY33849) and GoxA (accession number ADZ90918). With the sequence of GoxA a few more genes were detected, so this group of genes was selected since it included all the genes detected using LodA sequence as query.

Genes encoding LodB-like proteins were detected in a similar way, using as query LodB sequence (accession number AAY33850). In this case the BLASTP (E-value 1e-10) was limited only to those genomes containing lodA-like genes. In most cases, a single hit was obtained against a gene located next to the lodA-like gene and this was included in the group of lodB-like genes. In fewer cases several hits were obtained, but only the gene close to the lodA-like gene (which was the one with the highest score) was selected.

LodA-like proteins sequence and phylogenetic analysis All the protein sequences (168) selected in this study were aligned using the program clustal omega [33] available at http://www.ebi.ac.uk/Tools/msa/clustalo/.
Aligned sequences were incorporated into the program MEGA6 [21] to perform the phylogenetic analysis. Two different kind of analysis were performed. First, a tree was constructed using the Neighbor-Joining (NJ) method. In this method the distances between sequences were computed using the p-distance method and are in the units of the number of amino acid differences per site. The reliability of each node in the tree constructed was estimated using bootstrap analysis with 500 replicates.

A second analysis was performed using the Maximum Likelihood (ML) method. To select the most appropriate substitution model in the construction of the tree, the feature "Find Best DNA/Protein Model" incorporated in MEGA was used [34]. The model selected was Le and Gascue (LG) [35]. A discrete Gamma distribution was used to model evolutionary rate differences among sites (5 categories $(+\mathrm{G}$, parameter $=1,3855))$. The rate variation model allowed for some sites to be evolutionarily invariable $(+\mathrm{I}$, $4,2775 \%$ sites). Pairwise distances were estimated using a Jones-Taylor-Thorton (JTT) model. All positions with less than $95 \%$ site coverage were eliminated. That is, fewer than 5\% alignment gaps, missing data, and ambiguous bases were allowed at any position. The reliability of each node in the ML tree constructed was estimated using bootstrap analysis with 50 replicates.

The 168 proteins analyzed in this study were associated in groups containing five or more proteins. The criterion followed is that those groups should be supported by bootstrap analysis with higher than $70 \%$ reliability in the two phylogenetic analysis performed (NJ and ML).

\section{Availability of supporting data}

The phylogenetic tree with the 168 LodA-like proteins and its associated data matrix are available in TreeBASE database (Accession URL: http://purl.org/phylo/treebase/phylows/study/TB2:S17238). Other supporting data are included as Additional files 1, 2, 3, 4, 5, 6 and 7.

\section{Additional files}

Additional file 1: Table S1. Genes of the lodA family detected in IMG database as of January 2014.

Additional file 2: Table S2. lodB-like genes encoding putative flavoproteins detected in genomes containing a lodA-like gene. Additional genes located between both genes in some genomes are shown in red. Some genes that seem to be a lodB-like gene divided in two are shown in blue

Additional file 3: Figure S1. Phylogenetic relationships of LodA-like proteins in the group IB. The tree was constructed using the NeighborJoining method built in the MEGA6 program. The evolutionary distances were computed using the p-distance method and are in the units of the number of amino acid differences per site. Numbers at branches indicate bootstrap values $>70 \%$ for both Neighbor-Joining and Maximum Likelihood trees. Gammaproteobacteria are indicated in red, Alphaproteobacteria in light blue, Betaproteobacteria in dark blue and photosynthetic microorganisms in green. 
Additional file 4: Figure S2 Additional file 4. Phylogenetic relationships of LodA-like proteins in the group IC and ID. The tree was constructed using the Neighbor-Joining method built in the MEGA6 program. The evolutionary distances were computed using the p-distance method and are in the units of the number of amino acid differences per site. Numbers at branches indicate bootstrap values $>70 \%$ for both Neighbor-Joining and Maximum Likelihood trees. Gammaproteobacteria are indicated in red, Alphaproteobacteria in light blue and Betaproteobacteria in dark blue. Asterisks indicate those operons containing a small gene between the lod $A$ and lodB-like genes.

Additional file 5: Figure S3. Phylogenetic relationships of LodA-like proteins in the group III. The tree was constructed using the NeighborJoining method built in the MEGA6 program. The evolutionary distances were computed using the $p$-distance method and are in the units of the number of amino acid differences per site. Numbers at branches indicate bootstrap values $>70 \%$ for both Neighbor-Joining and Maximum Likelihood trees. Gammaproteobacteria are indicated in red, Alphaproteobacteria in light blue, Betaproteobacteria in dark blue and photosynthetic microorganisms in green. Asterisks indicate that the protein shows the conserved domain pfam00199.

Additional file 6: Figure S4. Phylogenetic relationships of LodA-like proteins in the group IV. The tree was constructed using the NeighborJoining method built in the MEGA6 program. The evolutionary distances were computed using the p-distance method and are in the units of the number of amino acid differences per site. Numbers at branches indicate bootstrap values $>70 \%$ for both Neighbor-Joining and Maximum Likelihood trees. Alphaproteobacteria are indicated in light blue.

Additional file 7: Figure S5. Phylogenetic relationships of LodA-like proteins detected in fungi (Group V). The tree was constructed using the Neighbor-Joining method built in the MEGA6 program. The evolutionary distances were computed using the $\mathrm{p}$-distance method and are in the units of the number of amino acid differences per site. Numbers at branches indicate bootstrap values $>70 \%$ for both Neighbor-Joining and Maximum Likelihood trees.

\section{Abbreviations}

LAO: L-amino acid oxidase; CTQ: Cysteine tryptophylquinone;

QHNDH: Quinohemoprotein amine dehydrogenase; BLAST: Basic Local Alignment Search Tool; ML: Maximum Likelihood; NJ: Neighbor-Joining.

\section{Competing interests}

The authors declare that they have no competing interests.

\section{Authors' contributions}

ASA conceived and designed the study, carried out the analysis, and drafted the manuscript. JCCB, MDCV and PLE participated in data analysis and revision of the manuscript. All authors read and approved submission of the manuscript.

\section{Acknowledgments}

This work has been supported by grants BIO2010-15226 (Ministerio de Ciencia e Innovación, Spain) and 11867/PI/09 (Fundación Séneca, CARM, Spain) (Co-financed by the European Commission, FEDER funds).

\section{Received: 25 November 2014 Accepted: 9 March 2015}

\section{Published online: 24 March 2015}

\section{References}

1. Hossain G, Li J, Shin H, Du G, Liu L, Chen J. L-Amino acid oxidases from microbial sources: types, properties, functions, and applications. Appl Microbiol Biotechnol. 2014;98:1507-15.

2. Yu Z, Qiao H. Advances in non-snake venom l-amino acid oxidase. Appl Biochem Biotechnol. 2012;167:1-13.

3. Du XY, Clemetson KJ. Snake venom L-amino acid oxidases. Toxicon. 2002:40:659-65.

4. Pollegioni L, Motta P, Molla G. L-Amino acid oxidase as biocatalyst: a dream too far? Appl Microbiol Biotechnol. 2013;97:9323-41.

5. Gomez D, Lucas-Elio P, Sanchez-Amat A, Solano F. A novel type of lysine oxidase: L-lysine-epsilon-oxidase. Biochim Biophys Acta. 2006;1764:1577-85.
6. Okazaki S, Nakano S, Matsui D, Akaji S, Inagaki K, Asano Y. X-Ray crystallographic evidence for the presence of the cysteine tryptophylquinone cofactor in L-lysine-E-oxidase from Marinomonas mediterranea. J Biochem. 2013;154:233-6.

7. Satoh A, Kim JK, Miyahara I, Devreese B, Vandenberghe I, Hacisalihoglu A, et al. Crystal structure of quinohemoprotein amine dehydrogenase from Pseudomonas putida. Identification of a novel quinone cofactor encaged by multiple thioether cross-bridges. J Biol Chem. 2002;277:2830-4.

8. Davidson VL. Protein-derived cofactors. Expanding the scope of post-translational modifications. Biochem. 2007:46:5283-92.

9. Chacon-Verdu M, Gomez D, Solano F, Lucas-Elio P, Sanchez-Amat A. LodB is required for the recombinant synthesis of the quinoprotein Hysine- $\varepsilon$-oxidase from Marinomonas mediterranea. Appl Microbiol Biotechnol. 2014;98:2981-9.

10. Gomez D, Lucas-Elio P, Solano F, Sanchez-Amat A. Both genes in the Marinomonas mediterranea lod $A B$ operon are required for the expression of the antimicrobial protein lysine oxidase. Mol Microbiol. 2010;75:462-73.

11. Mai-Prochnow A, Lucas-Elio P, Egan S, Thomas T, Webb JS, Sanchez-Amat A, et al. Hydrogen peroxide linked to lysine oxidase activity facilitates biofilm differentiation and dispersal in several Gram-negative bacteria. J Bacteriol. 2008;190:5493-501.

12. Lucas-Elio P, Goodwin L, Woyke T, Pitluck S, Nolan M, Kyrpides NC, et al. Complete genome sequence of the melanogenic marine bacterium Marinomonas mediterranea type strain (MMB-1 ${ }^{\top}$ ). Stand Genomic Sci. 2012;6:63-73.

13. Campillo-Brocal JC, Lucas-Elio P, Sanchez-Amat A. Identification in Marinomonas mediterranea of a novel quinoprotein with glycine oxidase activity. MicrobiolOpen. 2013;2:684-94.

14. Espinosa E, Marco-Noales E, Gomez D, Lucas-Elio P, Ordax M, Garcias-Bonet N, et al. Taxonomic study of Marinomonas strains isolated from the seagrass Posidonia oceanica, with descriptions of Marinomonas balearica sp. nov. and Marinomonas pollencensis sp. nov. Int I Syst Evol Microbiol. 2010;60:93-8.

15. Lucas-Elio P, Gomez D, Solano F, Sanchez-Amat A. The antimicrobial activity of marinocine, synthesized by Marinomonas mediterranea, is due to hydrogen peroxide generated by its lysine oxidase activity. J Bacteriol. 2006;188:2493-501.

16. Molina-Quintero LR, Lucas-Elio P, Sanchez-Amat A. Regulation of the Marinomonas mediterranea antimicrobial protein lysine oxidase by L-lysine and the sensor histidine kinase PpoS. Appl Environ Microbiol. 2010;76:6141-9.

17. Chacon-Verdu MD, Campillo-Brocal JC, Lucas-Elio P, Davidson VL, Sanchez-Amat A. Characterization of recombinant biosynthetic precursors of the cysteine tryptophylquinone cofactors of L-lysine-epsilon-oxidase and glycine oxidase from Marinomonas mediterranea. Biochim Biophys Acta 2015, http.//dx.doi.org/ 10.1016/j.bbapap.2014.12.018

18. Finn RD, Bateman A, Clements J, Coggill P, Eberhardt RY, Eddy SR, et al. Pfam: the protein families database. Nucleic Acids Res. 2014;42:D222-30.

19. Marchler-Bauer A, Zheng C, Chitsaz F, Derbyshire MK, Geer LY, Geer RC, et al. CDD: conserved domains and protein three-dimensional structure. Nucleic Acids Res. 2013;41:D348-52

20. Whittaker CA, Hynes RO. Distribution and evolution of von Willebrand/ Integrin A domains: Widely dispersed domains with roles in cell adhesion and elsewhere. Mol Biol Cell. 2002;13:3369-87.

21. Tamura K, Stecher G, Peterson D, Filipski A, Kumar S. MEGA6: Molecular evolutionary genetics analysis Version 6.0. Mol Biol Evol. 2013;30:2725-9.

22. James SG, Holmstrom C, Kjelleberg S. Purification and characterization of a novel antibacterial protein from the marine bacterium D2. Appl Environ Microbiol. 1996;62:2783-8.

23. Lucas-Elio P, Hernandez P, Sanchez-Amat A, Solano F. Purification and partial characterization of marinocine, a new broad-spectrum antibacterial protein produced by Marinomonas mediterranea. Biochim Biophys Acta. 2005;1721:193-203.

24. Yu M, Wang J, Tang K, Shi X, Wang S, Zhu WM, et al. Purification and characterization of antibacterial compounds of Pseudoalteromonas flavipulchra JG1. Microbiol. 2012;158:835-42.

25. Chen WM, Lin CY, Chen CA, Wang JT, Sheu SY. Involvement of an I-amino acid oxidase in the activity of the marine bacterium Pseudoalteromonas flavipulchra against methicillin-resistant Staphylococcus aureus. Enzyme Microb Technol. 2010;47:52-8.

26. Gomez D, Espinosa E, Bertazzo M, Lucas-Elio P, Solano F, Sanchez-Amat A. The macromolecule with antimicrobial activity synthesized by Pseudoalteromonas luteoviolacea strains is an L-amino acid oxidase. Appl Microbiol Biotechnol. 2008;79:925-30. 
27. Cress BF, Erkert KA, Barquera B, Koffas MAG. Draft genome sequence of Pseudoalteromonas luteoviolacea strain B (ATCC 29581). Genome Announc. 2013;1 (2):e00048-13.

28. Chen WM, Lin CY, Young CC, Sheu SY. Rheinheimera aquatica sp. nov., an antimicrobial activity producing bacterium isolated from freshwater culture pond. J Microbiol Biotechnol. 2010;20:1386-92.

29. Chen WM, Lin CY, Sheu SY. Investigating antimicrobial activity in Rheinheimera sp. due to hydrogen peroxide generated by L-lysine oxidase activity. Enzyme Microb Technol. 2010;46:487-93.

30. Gupta HK, Gupta RD, Singh A, Chauhan NS, Sharma R. Genome sequence of Rheinheimera sp. strain A13L, isolated from Pangong Lake, India. J Bacteriol. 2011;193:5873-4.

31. McWilliam H, Li W, Uludag M, Squizzato S, Park YM, Buso N, et al. Analysis Tool Web Services from the EMBL-EBI. Nucleic Acids Res. 2013;41:W597-600.

32. Markowitz VM, Chen IM, Chu K, Szeto E, Palaniappan K, Pillay M, et al. IMG/ M 4 version of the integrated metagenome comparative analysis system. Nucleic Acids Res. 2014:42:D568-73.

33. Sievers F, Wilm A, Dineen D, Gibson TJ, Karplus K, Li W, et al. Fast, scalable generation of high-quality protein multiple sequence alignments using Clustal Omega. Mol Syst Biol. 2014;7:539.

34. Hall BG. Building phylogenetic trees from molecular data with MEGA. Mol Biol Evol. 2013:30:1229-35.

35. Le SQ, Gascuel O. An improved general amino acid replacement matrix. Mol Biol Evol. 2008;25:1307-20.

\section{Submit your next manuscript to BioMed Central and take full advantage of:}

- Convenient online submission

- Thorough peer review

- No space constraints or color figure charges

- Immediate publication on acceptance

- Inclusion in PubMed, CAS, Scopus and Google Scholar

- Research which is freely available for redistribution 